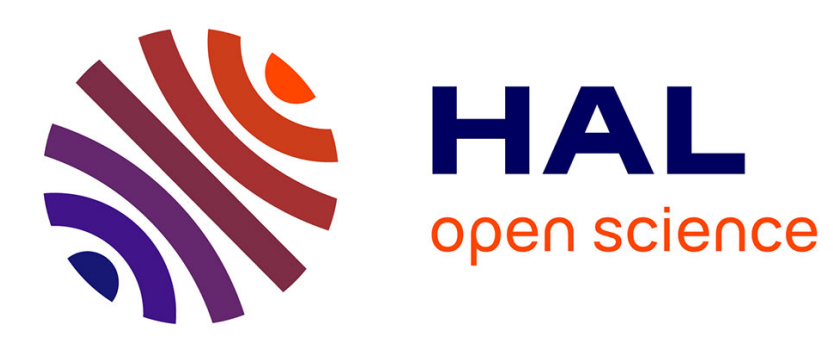

\title{
Enhanced spread in time on-off keying technique for dense Terahertz nanonetworks
}

Hakim Mabed

\section{To cite this version:}

Hakim Mabed. Enhanced spread in time on-off keying technique for dense Terahertz nanonetworks. Symposium on Computers and Communications, Jul 2017, Heraklion, Crete, Greece. hal-02472581

\section{HAL Id: hal-02472581 \\ https://hal.science/hal-02472581}

Submitted on 10 Feb 2020

HAL is a multi-disciplinary open access archive for the deposit and dissemination of scientific research documents, whether they are published or not. The documents may come from teaching and research institutions in France or abroad, or from public or private research centers.
L'archive ouverte pluridisciplinaire HAL, est destinée au dépôt et à la diffusion de documents scientifiques de niveau recherche, publiés ou non, émanant des établissements d'enseignement et de recherche français ou étrangers, des laboratoires publics ou privés. 


\title{
Enhanced Spread in Time On-Off Keying Technique for Dense Terahertz Nanonetworks
}

\author{
Hakim Mabed \\ Femto-ST institutes \\ University of Bourgogne Franche-Comté \\ Montbéliard, France \\ Email: hmabed@femto-st.fr
}

\begin{abstract}
Nanotechnology becomes reality paving the way for many new applications. In nanonetwork system, each nanosized device is equipped with limited capabilities and is dedicated to a basic task but the combination of the numerous devices actions results in high-level functions. In this context, large number of devices concentrated in a limited area must exchange data using wireless links. Spread in Time On-Off Keying (TS-OOK) protocol was proposed as a technique to share the radio channel over the different terahertz nano-devices. TS-OOK is based on a Femtosecond-Long pulse modulation where communication data are sent using a sequence of pulses interleaved by a constant duration randomly selected. In this paper we provide a critical analysis of the TS-OOK approach. We prove that the TS-OOK is not adaptive against the traffic load variation and induces an imbalance between the active nodes. This inequity is due to the dependency of a communication quality on the randomly chosen symbol rate. We propose a dynamic TS-OOK modulation approach, called SRH-TSOOK (Symbol rate Hopping TSOOK), where the duration between two consecutive pulses of the same transmission follows a pseudo-random sequence. We show that this approach performs better than the standard protocol when the number of active nodes increases and guarantees a better distribution of the channel capacity over the active communications. For instance, while the throughput of a communication within a TS-OOK protocol may falls bellow $10^{5} \mathrm{frames} / \mathrm{s}$ with 300 active nodes, the throughput in the SRH-TSOOK protocol stabilizes around $20^{7}$ frames $/ \mathrm{s}$ for all the active communications. The comparison is made on the basis of probabilistic analysis allowing a numerical and accurate evaluation of the protocols performance.
\end{abstract}

\section{INTRODUCTION}

Nanotechnology is moving forward in many domains. The objective is the fabrication of nano-size devices dedicated to a specific basic task such as identification [1], computing [2], sensing [3]. One of the promising applications is the programmable material [4], where a set of nano-scale robots change their positions in order to reach a given desired shape. To represent the physical aspect of a complex object, the nano-robots should be close enough to mask the empty spaces. Therefore a high number of nano-devices could be concentrated in the same area. In programmable material application, the size of robot is less than a cubic millimeter and the built objects can include billion of robots located in an area of $1 \mathrm{~m}^{3}$. A further application is the Wireless nano sensor network [5]. In this case, nano devices, which may be chemical particles, sense the local area and collaborate in order to provide accurate measurement of the environment.
In such systems, the main challenge is to conceive highly distributed and effective applications allowing the collaboration of numerous nodes. In other words, the question is to improve the communication procedures and allow faster data exchange between distant nodes without need for a physical contact. Therefore the use of wireless communications technology appears as a suitable solution. However, the use of traditional frequency bands is not convenient since the radio antenna size should be at the same scale as the used wavelength. Graphene based-antenna [6] enable the exploitation of Terahertz band [7] while accommodating the miniaturization requirements.

In [8], Jornet and Akyldiz proposed a femtosecond pulse based modulation technique for Terahertz communications called TS-OOK. In TS-OOK, radio channel corresponds to a sequence of time windows during which a symbol (0/1) is transmitted using an electromagnetic pulse of a duration $T p=100$ femtoseconds. The time duration between two consecutive symbols, $\beta$, of the same communication is negotiated (randomly chosen) during the communication establishment between the two nodes [9] and remains constant during all data transmission session. This proposition is similar to MAC protocol in Ultra Wide Band (UWB) technology [10]. Except that the electrical pulse in UWB is larger with picosecond range and the covering range is about 10 meters against 1 meter for Terahertz nanonetworks.

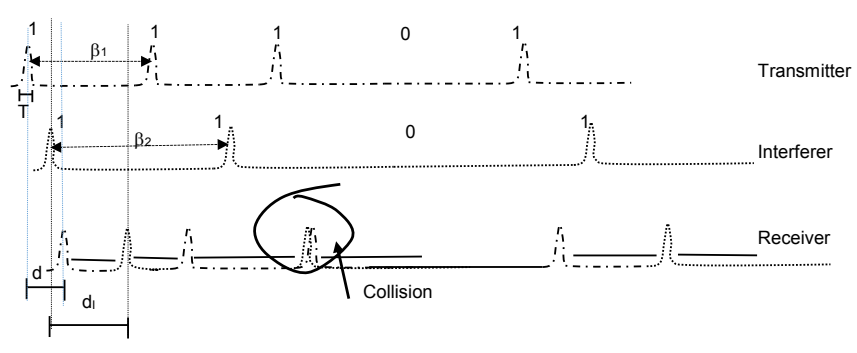

Fig. 1. TS-OOK modulation: $\beta_{1}$ and $\beta_{2}$ correspond to the inter-symbol period for the transmitter and the jammer nodes. The useful and jamming signals are received with a delay depending on the distance between the nodes.

To reduce the collision probability between communications, two solutions was proposed [9]. First, only 1-symbol is coded by a pulse while 0 -symbol is represented by a silence. 
Therefore the interfering impact of every communication is theoretically divided by 2 . This advantage is better explored in [11] using a re-encoding procedure where the transmitted symbols are more likely 0 than 1 . Secondly, the symbol rate, $\beta$, of a given communication is selected among a list of coprime numbers for example: 1001, 1002, 1003, 1007, etc. Therefore if a collision occurs at a receiver (see Fig. 1), the future collision between the same nodes could not occurs before a period of $\beta_{1} \times \beta_{2}$, where $\beta_{1}$ and $\beta_{2}$ are respectively the symbol rate of transmitter and jammer node.

The remaining paper is organized as follow. In section 2 , we give a critical analysis of TS-OOK protocol where we underline its major disadvantages. Then in section 3, we present our Symbol Rate Hopping TSOOK approach. In section 4, we compare the standard and proposed approaches using probabilistic modeling of Bit Error Rate, Frame Error Rate and the MAC throughput of a given communication. In the last section, we conclude this work and give the major perspectives.

\section{CRITICAL ANAlysis OF TS-OOK}

In TS-OOK, a communication, $i$, is carried by pulses (or silences for 0-symbol transmission) interleaved by a regular period. Let $T s_{i}$ the inter-symbol duration of the communication $i$ and $T p$ the pulse duration (100 femtoseconds). Before data exchange, the transmitter sends a communication request to the destination(s) using a control channel known by its constant inter-symbol duration $T s_{0}$. In the communication request, the transmitter announces the value of $T s_{i}$ that will be used. Each received pulse allows to the receiver to synchronize with the transmitter to take into account changes on the communication parameters (distance, air composition, etc). The symbol rate $\beta_{i}$ of the communication $i$ is determined by the ratio $T s_{i} / T p$. The symbol rate is selected among a list of coprime numbers belonging to the interval $\left[\beta_{\min }, \beta_{\max }\right]$. $\beta_{\min }$ and $\beta_{\max }$ represent respectively the upper and the lower data rate tolerated by the protocol. To best of our knowledge, the number of coprime numbers belonging to a given interval cannot be computed by numerical formulation. In fig. 2, we depict the evolution of the number of coprime values according to the evolution of the $\left[\beta_{\min }, \beta_{\max }\right]$ range.

The distributed approach of the TS-OOK protocol, leads to select the symbol rate randomly during the communication establishment in order to spread the co-channel risks over the active nodes. However, a high symbol rate may be selected inducing a low throughput even though the radio channel is free.

\section{A. co-channel probability}

According to TS-OOK mode, the probability that two active nodes $\mathrm{T}$ (transmitter) and I (jammer) be synchronized in reception at $R$ (receptor) node is equal to the probability that:

- the two nodes use the same symbol rate $\beta=T s / T p$ and

- $\left(t_{T}+d_{T}\right) \% T s=\left(t_{I}+d_{I}\right) \% T s \pm T_{p}$

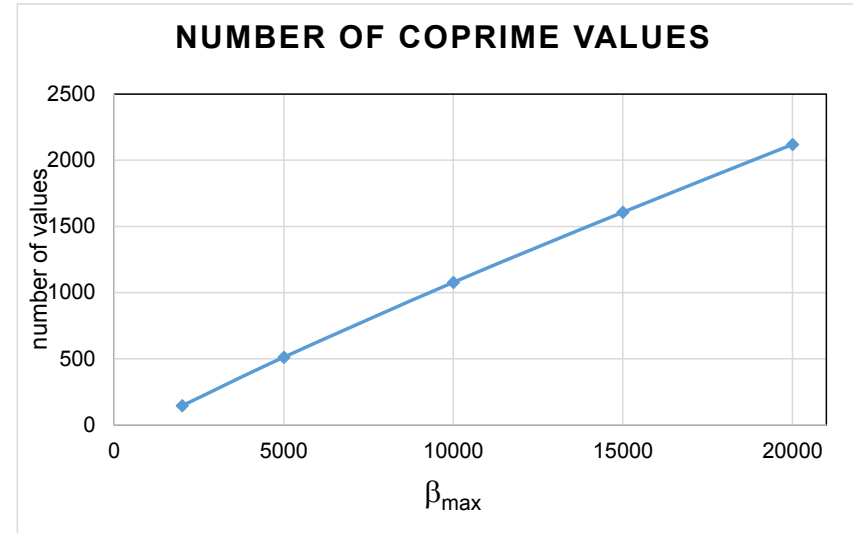

Fig. 2. Evolution of the number of corpime values according to $\beta_{\max }$. $\beta_{\min }=1000$

Where $t_{T}$ and $d_{T}$ (resp. $t_{I}$ and $d_{I}$ ) are the beginning time and the path duration (between the sender and $R$ ) of $T$ signal (resp. I signal).

Figures 1 shows an example of the reception of two communications originating from two source nodes. The second condition refers to the probability that the interfering pulse overloads the transmitter pulse.

Therefore the probability that the transmitter and jammer pulses be received systematically at the same time by a receiver (co-channel interference) is equal to:

$$
P[T, I \text { co-channel at } R]=\frac{1}{\text { nbcoprime }} \times \frac{1}{\beta}
$$

In equation (1), nbcoprime represents the number of coprime values available in the interval $\left[\beta_{\min }, \beta_{\max }\right]$. For instance with $\left[\beta_{\min }, \beta_{\max }\right]=[1000,5000]$ and Transmitter symbol rate $\beta=2089$, co-channel probability is equal to $\frac{1}{511} \times \frac{1}{2089}=9.36 \times 10^{-7}$.

\section{B. Bit Error Rate in TS-OOK}

TS-OOK allows to adjust the system to the application requirements by setting the range of $\left[\beta_{\min }, \beta_{\max }\right]$ interval. However, increasing the interval range obviously increases the number of coprime values but increases also the disparity of the symbol rate between communications using a value of $\beta \approx \beta_{\min }$ and those with $\beta \approx \beta_{\max }$. Communications using low symbol rate benefit of a better data rate than those using high symbol rate. In the other side, it is expected that when the number of interfering nodes increases, communications with lower symbol rate are more subject to collisions.

Indeed with a density of 1 node by $1 \mathrm{~mm}^{2}$, the system may reach a population of $10^{6}$ nodes in $1 \mathrm{~m}^{2}$ that largely exceeds the thousand of available symbol rates. The fact that nodes are not all active at the same time offers a protection against co-channel collisions between pair of communications, but the increase of active nodes number increase the observed BER.

Moreover, when nodes are located in a 3D area (programmable material case for instance), the number of nodes 
concentrated in a limited area increases exponentially and hence amplifies the collision probability.

Let $R$ the reference node that listens to the communication transmitted by the node $T$ and let $N$ the number of surrounding nodes (nodes which signal can be received by $R$ ). Let nbcoprime the number of available coprime values. We assume that communications arrivals follow a Poisson distribution of parameter $\lambda$ (corresponding to the average number of new communications in a second) and we assume that the communication duration follows an exponential distribution with a mean $\frac{1}{\mu}$. The conjunction of the two distributions (arrivals and communication durations) makes that the number of active nodes follows a Poisson distribution with a parameter $\theta=\lambda \times \mu$. The Poisson distribution represents a good model [12], [13], [14] for arrival phenomena when the number of communication sources (nodes) is high enough, which is the case for the studied scenarios (the number $N$ ). Indeed, the higher the number of communication sources is, more relevant the assumption about the inter-dependence of communications arrivals is. However the assumption concerning the communication duration neglects the effect of the symbol rate selection and the interference impact on the communication. The interference aspect can however be ignored by considering not reliable communications where the lost frames are not retransmitted and by assuming a fix error code mechanism.

The probability that the number of active nodes around the node $R$ be equal to $k$ is expressed by the following formula:

$$
\begin{aligned}
P[\mathrm{k} \text { active nodes }] & =\frac{\theta^{k}}{k !} \times\left[\sum_{i=1}^{\infty} \frac{\theta^{i}}{i !}\right]^{-1} \\
& =\frac{\theta^{k}}{k !} \times e^{(-\theta)}
\end{aligned}
$$

When the listened communication uses the symbol rate $\beta$, the probability that a received symbol by $R$ be in collision with a symbol transmitted by another active communication, $I$, is equal to $\frac{1}{\beta}$. With $p 1$ the probability that transmitted symbol be 1 (a pulse and not a silence), the probability that a symbol of the listened communication be interfered by a pulse of the communication $I$ is expressed by:

$$
P[I \text { interferes with } T]=\frac{p 1}{\beta}
$$

The probability $p 1$ could be less than 0.5 if Low-weight Channel coding [11] is used. We assume that the receptor uses the correlation-based energy detector extending the reception window to $2 \times T_{p}$. We conclude that:

$$
P[I \text { interferes with } T]=2 \times \frac{p 1}{\beta}
$$

In the following we consider that $p 1=0.5$.

The joint action of the random selection of the symbol rates among coprime values and the randomness of communications arrivals leads to a uniform distribution in time of the interfering pulses. In the following, we assume that every concurrent communication is a potential jamming signal, which means that the signal powers are ignored. Therefore, the probability that a symbol of the listened communication be interfered by a communication transmitted by one of the $k$ active nodes can be expressed by:

$$
P[\exists I \text { interferes } T / k \text { active nodes }]=\sum_{i=0}^{k}\left(\frac{\beta-1}{\beta}\right)^{i} \times \frac{1}{\beta}
$$

The probability that a received symbol by $R$ be interfered by at least another communication (whatever is the number of active nodes) is equal to:

$$
\begin{aligned}
& B E R \\
& =\sum_{k=1}^{\infty} P[\exists I \text { interferes } T / k \text { active nodes }] \times P[k \text { active nodes }]
\end{aligned}
$$

Which gives the BER of a given communication:

$$
B E R=\sum_{k=1}^{\infty} \sum_{i=0}^{k}\left(\frac{\beta-1}{\beta}\right)^{i} \times \frac{1}{\beta} \times \frac{\theta^{k}}{k !} \times e^{(-\theta)}
$$

The observation of the BER formula reveals two main points. First the BER is closely linked to the symbol rate used by the communication. When the $\beta$ is low, the value of the BER increases. We deduce that low $\beta$ provides a better symbol throughput but increases the data loss when the number of active nodes augments. Secondly, the random selection of the symbol rate produces an unequal treatment between the different communications. In addition, the use of high symbol rate when the radio channel is free (low number of active nodes) is not relevant. In the next section we propose an enhanced TS-OOK protocol allowing to overcome these drawbacks.

\section{Symbol RATE Hopping TS-OOK}

To address the disadvantages of TS-OOK protocol we were inspired by the frequency hopping technique used in FDTDMA networks [15]. In Slow Frequency Hopping technique, the interference are repartitioned using a pseudo-random sequence. Every transmitter hops over the different available frequencies using pseudo-random sequences [16], [17]. In place of hopping over different frequencies as in FD-TDMA technology, in our SRH-TSOOK protocol, transmitters hop over different symbol rates using a pseudo-random sequence known by both the transmitter and the receiver. The random sequence guarantees that the different symbol rates are used uniformly. Consequently, all active communications has the same transmission conditions expressed by the same average of BER and data rate. The Symbol Rate hopping aims to repartee the observed interferences over the concurrent communications. The operation mode of the Symbol Rate hopping TS-OOK method is depicted in Fig. 3.

The idea is to change the symbol rate of a communication after a regular number of symbols corresponding to the transmission of a MAC frame. The repartition of the interference depends on the shortness of this delay. The use of symbol rate hopping eliminates the effect of coprime values on reducing 

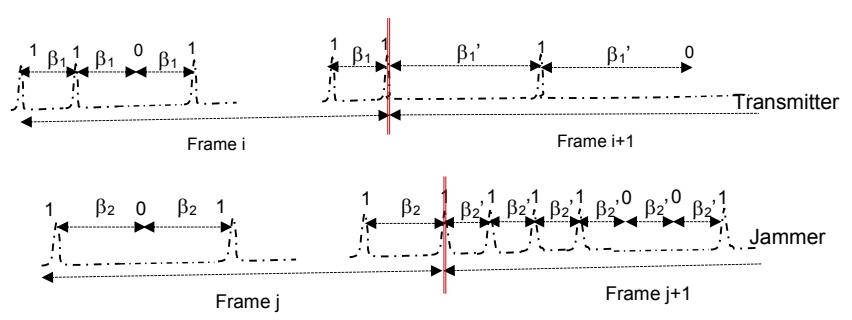

Fig. 3. Symbol Rate Hopping: after each frame, a new symbol rate $\beta$ is generated and used to transmit the next frame.

the pair-nodes interferences. However, this feature is relatively less important than the reduction of the interference sum.

Many random generators exist in literature [18], [19] that can mainly expressed as a recursive function $R N D$ :

$$
r_{\text {current }}=R N D\left(r_{\text {current }}\right)
$$

$r_{\text {current }}$ represents the current random value. The selection of the $R N D$ function should take into account the power limitation of the nano/micro-machines. A solution such as Linear Congruential Generator [20] could be then considered.

During the communication establishment, the transmitter and the receiver agree on the initial $r_{\text {current }}$ value called seed using the control channel $\left(T s_{0}\right)$. The sequence of numbers $r_{\text {current }}$ is then computed without exchange between receiver and transmitter based on the deterministic function $R N D$. After each exchanged MAC frame, the transmitter and the receiver use the new $r_{\text {current }}$ (eq. 8) to calculate the next symbol rate as follow:

$$
\beta=\frac{r_{\text {current }} \times\left(\beta_{\max }-\beta_{\text {min }}\right)}{R N D M A X}+\beta_{\text {min }}
$$

RNDMAX represents the highest value returned by the RND function.

\section{Performance AnAlysis of SRH-TSOOK}

Now, we start studying the contribution of the SRH-TSOOK on the performance of the nano-networks. The co-channel probability, BER, FER and data throughput are taken as metrics to compare the standard and the proposed MAC protocols.

\section{A. co-channel interference in SRH-TSOOK}

Co-channel interference occurs when two communications are systematically in collision, which means that pulses sent by $T$ to $R$ always arrive at the same time that the interfering pulses sent by $I$. In SRH-TSOOK, co-channel probability is equal to the probability that the two communications use the same seed (the initial $r_{c}$ urrent) of the pseudorandom sequence. Since the seed is selected in the interval $[O, R N D M A X]$, the co-channel interference is equal to $1 / R N D M A X$. With $R N D M A X=2^{32}$, the co-channel interference probability is then well below the co-channel probability in standard TS-OOK protocol given in equation 1.

\section{B. BER in SRH-TSOOK}

Since every communication uses sequentially the different available symbol rates in random way, the BER of a communication is equal to the average BER obtained using each symbol rate; which corresponds to the following expression:

$$
B E R=\frac{\sum_{\beta=\beta_{\min }}^{\beta_{\max }} \sum_{k=1}^{\infty} \sum_{i=0}^{k}\left(\frac{\beta-1}{\beta}\right)^{i} \times \frac{1}{\beta} \times \frac{\theta^{k}}{k !} \times e^{(-\theta)}}{\beta_{\max }-\beta_{\min }}
$$

In Fig. 4, we present the communication BER according to the average number of active nodes. We compare the estimated BER of 6 communications using different constant symbol rates with the estimated BER of a communication using SRHTSOOK protocol. We remind that the quality of a given communication in the standard TS-OOK protocol varies according to the selected symbol rate while this quality is smoothed over all the communications in the SRH-TSOOK approach. Figure shows that SRH-TSOOK allows to bring the BER to average values for all communications while the BER reaches high values in standard TS-OOK for communications using a low symbol rate $(\beta=1000)$. For instance, under 900 concurrent communications, a communication with symbol rate equal to 1000 suffers from a BER that exceeds the $60 \%$, while the BER is less than 30\% when the Symbol Rate Hoping is used for all communications. By contrast, in standard TSOOK, communication that uses the symbol rate 5000 has a better BER (less than $10 \%$ with a number of active nodes bellow 600). However by using a high symbol rate, we expect that the data rate of such communication will be low. SRHTSOOK allows to obtain better data rate without extremely increases the BER value.

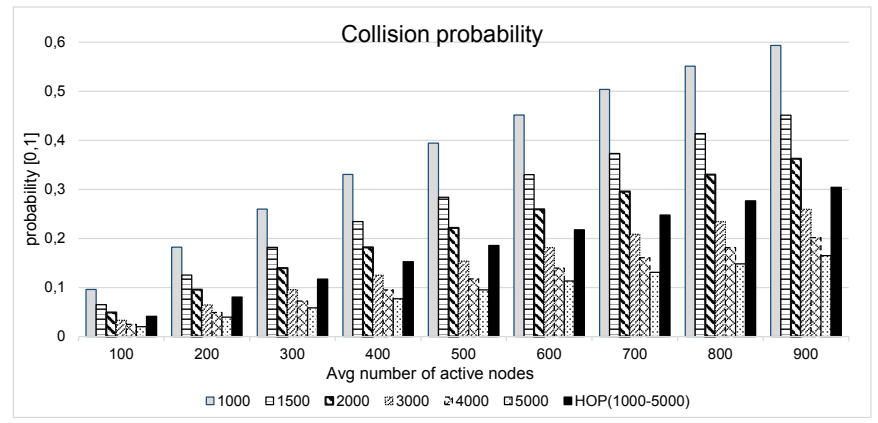

Fig. 4. Comparison between the BER obtained by TS-OOK protocol for 6 communications using different fixed symbol rate $(1000,1500,2000,3000,4000,5000)$ with the BER obtained by SRH-TSOOK protocol for any communication.

\section{FER in SRH-TSOOK}

The precedent section gives the BER of a communication using the SRH-TSOOK protocol. However, to better evaluate the quality of a communication the transmission error should be estimated in higher level to take into account detection and correction error codes [21]. Let evaluate now the error rate at MAC frame level. Let $n$ the number of bits composing 
a MAC frame (data + detection/correction Error code). We assume that the used error code offers a capability [22] of $m$, which means that the error code is able to fix the received frame if the number of corrupted bits does not exceed $m$. The number of corrupted bits (number of collided bits) in a MAC frame of size $n$ follows a Binomial distribution with a parameter $B E R$ (equation 7 or 10 according to used symbol rate allocation). Consequently, the probability that the number of corrupted bits be equal to $x$ is:

$$
P[x \text { corrupted bits }]=C_{n}^{x} \times B E R^{x} \times(1-B E R)^{n-x}
$$

The probability that the number of corrupted bits be higher or equal to $m$ is:

$$
\begin{aligned}
P & {[\text { corrupted bits } \geq m] } \\
& =\sum_{x=m}^{n} C_{n}^{x} \times(B E R)^{x} \times(1-B E R)^{n-x}
\end{aligned}
$$

Where $C_{n}^{x}=n ! / k ! \times(n-k)$ ! corresponds to the Binomial coefficient. Since the error code capability is limited to $m$ errors, we deduce that the Frame Error Rate is equal to:

$$
F E R=\sum_{x=m}^{n} C_{n}^{x} \times(B E R)^{x} \times(1-B E R)^{n-x}
$$

Fig. 5 represents the FER variation of a given communication according to the number of concurrent nodes and to the used symbol rate. The used MAC frame size is of 128 bits and $m$ is 20 . The figure shows that SRH-TSOOK method allows a more progressive evolution of the FER contrary to TS-OOK protocol where the FER suddenly increases with the evolution of the number of active nodes. For instance, when the symbol rate is equal to 1000 , the FER increases from $0 \%$ to $80 \%$ by varying the number of active nodes between 100 and 200 . Whereas the FER evolution does not exceed $10 \%$ with SRHTSOOK when the number of active nodes varies from 100 to 200. In the other side, while the standard TS-OOK presents certain communications with low FER $(\beta=5000)$ and other with high FER $(\beta=1000)$, SRH-TSOOK presents the same average FER for all the active communications.

\section{Data rate in SRH-TSOOK}

To estimate the impact of FER on the communication throughput (a lost frame is a retransmitted frame), we assume for simplification that frame acknowledgments are without errors and does not induce delay. The average number of frame retransmissions is equal to $1 /(1-F E R)$ times, where FER is determined using the equation 13 .

The frame transmission duration is equal to $n \times \beta \times T p$. Where $n$ is the frame size (in bits), $\beta$ is the symbol rate of the studied communication and $T p$ is the pulse duration. In case of SRH-TSOOK the $\beta$ value changes after each frame transmission. In case of standard TS-OOK, the data throughput of a given communication is equal to:

$$
\text { Throughput }=(1-F E R) /(n \times \beta \times T p) \text { Frames } / \mathrm{s}
$$

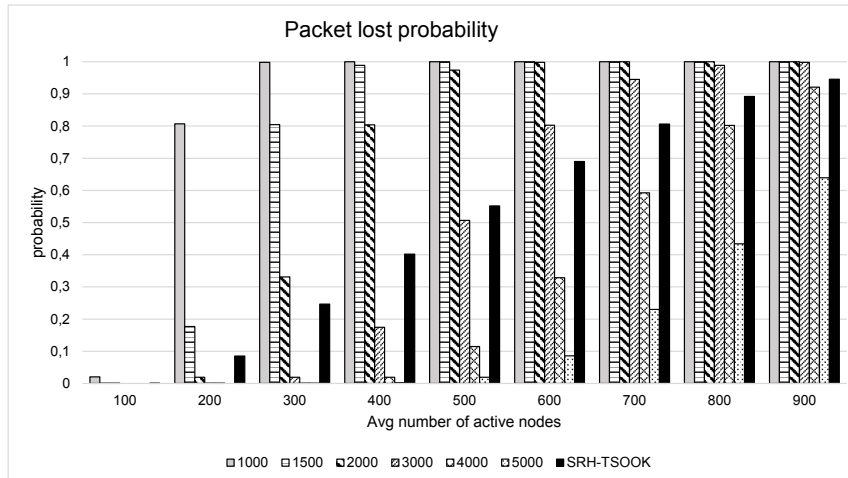

Fig. 5. Comparison between the FER obtained by TS-OOK protocol for 6 communications using respectively a symbol rate $(1000,1500,2000,3000,4000,5000)$ with the FER obtained by SRH-TSOOK method for any communication.

In case of SRH-TSOOK, the throughput is expressed as an average over available symbol rates.

$$
\begin{aligned}
& \text { Throughput } \\
& =\frac{\sum_{\beta=\beta_{\min }}^{\beta_{\max }}(1-F E R) /(n \times \beta \times T p)}{\left(\beta_{\max }-\beta_{\min }\right)} \text { Frames } / \mathrm{s}
\end{aligned}
$$

Fig. 6 allows to observe the evolution of throughput according the number of active nodes and used symbol rate. We observe clearly that SRH-TSOOK provides compromise between low and high symbol rate with a relatively good performances under low number and high number of concurrent communications. Contrary, TS-OOK presents an imbalance of the performance between communications using low symbol rate and those using high symbol rate. Whereas communications with low symbol rate perform well under weak load and give bad result under high load, communications with high symbol rate achieve the contrary. The imbalance that characterizes the standard TS-OOK makes that some communications are more subject to interference and therefore to data retransmission, which leads to a disproportion of energy consumption. Our proposed RSH-TSOOK mechanism guarantees the equal radio access conditions to all communications.

In Fig. 7, we give the comparison between the estimated Throughput of any communications using the RSH-TSOOK protocol and the worst throughput provided by the standard TS-OOK (when a random communication uses the worst symbol rate according to Fig. 6). The figure shows that standard TS-OOK performs badly when the traffic load increases while RSH-TSOOK displays a linear decrease according to traffic load. In addition when the traffic load is low, the randomness of symbol rate selection in standard TS-OOK protocol involves communications that do not benefit from the radio channel availability.

\section{CONClusion AND PERSPECTIVES}

In this paper we studied the Medium Access Control protocol in Terahertz based nanonetworks. We provided a critical 


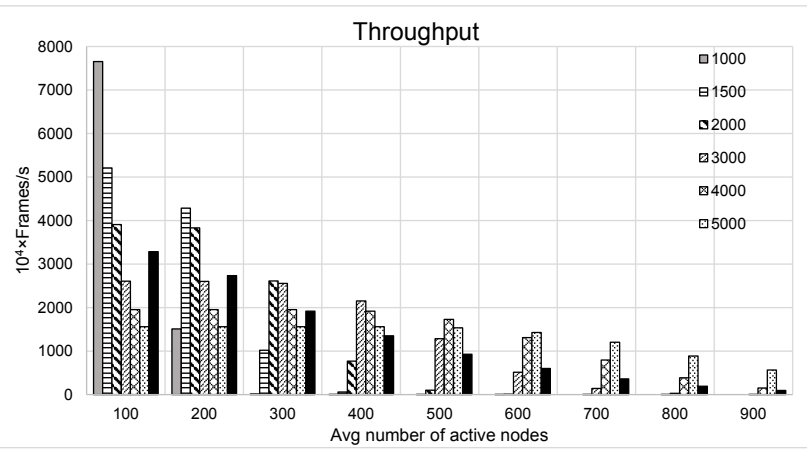

Fig. 6. Comparison between the throughput obtained by TS-OOK protocol for 6 communications using respectively a symbol rate $(1000,1500,2000,3000,4000,5000)$ with the throughput of any communication in SRH-TSOOK protocol.

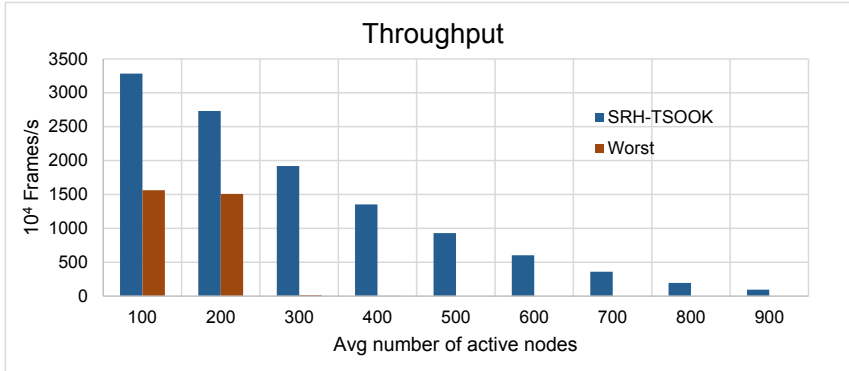

Fig. 7. Comparison between the lowest throughput obtained by a communication using standard TS-OOK with the throughput of any communication obtained using SRH-TSOOK method.

and original analysis of Spread in Time On-Off Keying (TSOOK) technique. This analysis shows that standard TS-OOK suffers of two major drawbacks. First, the random selection of the symbol rate induces imbalance in communications throughput between communication using the high symbol rate and those using low symbol rate. Secondly, the TS-OOK presents a lack of flexibility and adaptability against traffic load.

We proposed a Symbol Rate Hopping TS-OOK technique allowing to overcome these drawbacks. SRH-TSOOK is inspired by the Slow Frequency Hopping technique used in FDTDMA radio networks. The idea is to change regularly the symbol rate used by the communication in a pseudo-random way. This approach allows to spread the generated interference over the different communications and gives the same radio access conditions to all concurrent communications. The probabilistic analysis made in the paper proves the relevance of proposed techniques in terms of BER, FER and Throughput gain. In addition, the probabilistic analysis allows to clearly identify the impact of each parameter on the system behavior and allows to deal with big scenarios contrary to simulation analysis.

Some aspects of this analysis should be in-depth by introducing the signal powers. Indeed, in this paper we only studied the collision probability between sent pulses assuming that nodes are close enough to consider that collision induces certainly a symbol loss. However the interference depends also on the ratio between listened and interfering signals.

In addition, the flexibility of the SRH-TSOOK technique should be improved by adjusting the $\left[\beta_{\min }, \beta_{\max }\right]$ interval according to observed traffic load in the network. Therefore, when a low traffic is detected, communications can hop only on low symbol rate values to increase the throughput.

\section{REFERENCES}

[1] R. Pathak and S. Joshi, Multi-scale Modeling and Analysis of NanoRFID Systems on HPC Setup, Series Communications in Computer and Information Science, Vol. 40, pp 649-659, Springer, 2009.

[2] M. A. Bounouar, D. Drouin and F. Calmon, Towards nano-computing blocks using room temperature double-gate single electron transistors, IEEE 12th International Conference on New Circuits and Systems (NEWCAS), pp 325-328, IEEE, 2014.

[3] C. K. Sung, M. Egan, Z. Chen; I. B. Collings, Performance of Wireless Nano-Sensor Networks with Energy Harvesting, IEEE 79th Vehicular Technology Conference (VTC Spring), pp 1-5, IEEE, 2014.

[4] N. Boillot, D. Dhoutaut and J. Bourgeois, Using Nano-wireless Communications in Micro-Robots Applications, 1st ACM International Conference on Nanoscale Computing (NANOCOM), pp 1-9, ACM, 2014.

[5] I. F. Akyildiz and J. M. Jornet, Electromagnetic wireless nanosensor networks, Journal of Nano Communication Networks, N 1, pp 319, Elsevier, 2010

[6] X. Qin, J. Chen, C. Xie, N. Xu and J. Shi, A tunable THz dipole antenna based on graphene, IEEE MTT-S International Microwave Workshop Series on Advanced Materials and Processes for RF and THz Applications (IMWS-AMP), pp 1-3, IEEE, 2016.

[7] M. Ye. Ilchenko, S. E. Kuzmin, T. N. Narytnik and V. N. Radzikhovsky, Tranceiver for digital radiorelay system in terahertz frequency band, 23rd International Crimean Conference in Microwave and Telecommunication Technology (CriMiCo), pp 318-319, IEEE, 2013.

[8] J. M. Jornet and I. F. Akyildiz, Femtosecond-long Pulse-based Modulation for Terahertz Band Communication in Nanonetworks, IEEE Transactions on Communications, vol. 62, no. 5, pp 1742-1754, IEEE, 2014.

[9] J. M. Jornet, J. Capdevila-Pujol and J. Sole-Pareta, PHLAME: A Physical Layer Aware MAC Protocol for Electromagnetic Nanonetworks in the Terahertz Band, Journal of Nano Communication Networks, vol. 3, no. 1, pp. 74-81, Elsevier, 2012.

[10] M. S. I. M. Zin and M. Hope, A Review of UWB MAC Protocols, 6th Advanced International Conference on Telecommunications (AICT), pp 526-534, IEEE, 2010.

[11] J. M. Jornet, Low-weight Channel Codes for Error Prevention in Electromagnetic Nanonetworks in the Terahertz Band, 1st ACM International Conference on Nanoscale Computing and Communication (NANOCOM), ACM, 2014.

[12] D. Huo, Generalized Erlang-B formula for mobile and wireless radio channels, Global Telecommunications Conference, Communication Theory Mini-Conference, (GLOBECOM), pp 38-41, IEEE, 1995.

[13] M. D. Logothetis and I. D. Moscholios, Teletraffic models beyond Erlang, ELEKTRO, pp 10-15, IEEE, 2014.

[14] D. I. Kim, Multiple Access Performance of M-ary Orthogonal Balanced UWB Transmitted-Reference Systems, IEEE International Conference on Communications, pp 3933-3937, IEEE, 2008.

[15] P. Stuckmann, The GSM Evolution: Mobile Packet Data Services, John Wiley\&Sons Editions, 2003.

[16] D. Verhulst, M. Mouly and J. Szpirglas, Slow frequency hopping multiple access for digital cellular radiotelephone, IEEE Transactions on Vehicular Technology, Vol. 33, N 3, pp 179 - 190, IEEE, 1984.

[17] M. Chiani, A. Conti and O. Andrisano, Up-link analytical outage evaluation for slow frequency hopping mobile radio systems, IEEE International Conference on Communications (ICC), Vol. 3, pp 1922 1927, IEEE, 1999

[18] L. Blum, M. Blum and M. Shub, A Simple Unpredictable PseudoRandom Number Generator, SIAM Journal on Computing, Vol. 15, N 2, pp 364383, 1986.

[19] R. Impagliazzo, L. A. Levin and M. Luby, Pseudo-random Generation from One-way Functions,21th Annual ACM Symposium on Theory of Computing (STOC), pp 12-24, ACM, 1989. 
[20] P. L'Ecuyer, Tables of Linear Congruential Generators of Different Sizes and Good Lattice Structure, Mathematics of Computation. 68 (225), pp 249 260. doi:10.1090/S0025-5718-99-00996-5

[21] M. Franceschini, G. Ferrari and R. Raheli, LDPC Coded Modulation, Springer-Verlag Berlin, ISBN 10 3642088961, 2010.

[22] S.K. Chilappagari, B. Vasic and M.W. Marcellin, Guaranteed Error Correction Capability of Codes on Graphs, IEEE Information Theory and Applications Workshop, pp 50 - 55, IEEE, 2009. 